\title{
Potential role for LINAC-based stereotactic radiosurgery for the treatment of 5 or more radioresistant melanoma brain metastases
}

\author{
*Jessica M. Frakes, MD, ${ }^{1}$ Nicholas B. Figura, BS, ${ }^{2}$ Kamran A. Ahmed, MD, ${ }^{1}$ Tzu-Hua Juan, MPH, ${ }^{3}$ \\ Neha Patel, BS, ${ }^{2}$ Kujtim Latifi, PhD, ${ }^{1}$ Siriporn Sarangkasiri, MS, ${ }^{1}$ Tobin J. Strom, MD, ${ }^{1}$ \\ Prakash Chinnaiyan, MD, ${ }^{1,2}$ Nikhil G. Rao, MD, ${ }^{1,2}$ and Arnold B. Etame, MD, PhD ${ }^{2,4}$
}

Departments of ${ }^{1}$ Radiation Oncology, ${ }^{3}$ Biostatistics, and ${ }^{4}$ Neuro-Oncology, H. Lee Moffitt Cancer Center and Research Institute; and 2 University of South Florida, Morsani College of Medicine, Tampa, Florida

OBJECT Linear accelerator (LINAC)-based stereotactic radiosurgery (SRS) is a treatment option for patients with melanoma in whom brain metastases have developed. Very limited data are available on treating patients with $\geq 5$ lesions. The authors sought to determine the effectiveness of SRS in patients with $\geq 5$ melanoma brain metastases.

METHODS A retrospective analysis of metastatic melanoma treated with SRS in a single treatment session for $\geq 5$ lesions was performed. Magnetic resonance imaging studies were reviewed post-SRS to evaluate local control (LC). Disease progression on imaging was defined using the 2009 Response Evaluation Criteria in Solid Tumors (RECIST). Survival curves were calculated from the date of brain metastases diagnosis or the date of SRS by using the KaplanMeier (KM) method. Univariate and multivariate analysis (UVA and MVA, respectively) were performed using the Cox proportional-hazards model.

RESULTS The authors identified 149 metastatic brain lesions treated in 28 patients. The median patient age was 60.5 years (range 38-83 years), and the majority of patients (24 [85.7\%]) had extracranial metastases. Four patients $(14.3 \%)$ had received previous whole-brain radiotherapy (WBRT), and $11(39.3 \%)$ had undergone previous SRS. The median planning target volume (PTV) was $0.34 \mathrm{~cm}^{3}$ (range $0.01-12.5 \mathrm{~cm}^{3}$ ). Median follow-up was 6.3 months (range 1-46 months). At the time of treatment, $7 \%$ of patients were categorized as recursive partitioning analysis (RPA) Class I, $89 \%$ as RPA Class II, and 4\% as RPA Class III. The rate of local failure was $11.4 \%$. Kaplan-Meier LC estimates at 6 and 12 months were $91.3 \%$ and $82.2 \%$, respectively. A PTV volume $\geq 0.34 \mathrm{~cm}^{3}$ was a significant predictor of local failure on UVA (HR 16.1, 95\% Cl 3.2-292.6, $p<0.0001$ ) and MVA (HR 14.8, 95\% Cl 3.0-268.5, $p=0.0002$ ). Sixteen patients $(57.1 \%)$ were noted to have distant failure in the brain with a median time to failure of 3 months (range 1-15 months). Nine patients with distant failures received WBRT, and 7 received additional SRS. Median overall survival (OS) was 9.4 and 7.6 months from the date of brain metastases diagnosis and the date of SRS, respectively. The KM OS estimates at 6 and 12 months were $57.8 \%$ and $28.2 \%$, respectively, from the time of SRS treatment. The RPA class was a significant predictor of KM OS estimates from the date of treatment $(p=0.02)$. Patients who did not receive WBRT after SRS treatment had decreased OS on MVA (HR 3.5, 95\% CI 1.1-12.0, $p=0.03$ ), and patients who did not receive WBRT prior to SRS had improved OS (HR 0.11, 95\% Cl 0.02-0.53, $p=0.007$ ).

CONCLUSIONS Stereotactic radiosurgery for $\geq 5$ lesions appears to be effective for selected patients with metastatic melanoma, offering excellent LC. This is particularly important for patients as new targeted systemic agents are improving outcomes but still have limited efficacy within the central nervous system.

http://thejns.org/doi/abs/10.3171/2014.12.JNS141919

KEY WORDS stereotactic radiosurgery; LINAC; brain metastases; melanoma; oncology

ABBREVIATIONS DC = distant control; GTV = gross tumor volume; KM = Kaplan-Meier; LC = local control; LINAC = linear accelerator; MVA = multivariate analysis; OS = overall survival; PTV = planning target volume; RECIST = Response Evaluation Criteria in Solid Tumors; RPA = recursive partitioning analysis; RTOG = Radiation Therapy Oncology Group; SRS = stereotactic radiosurgery; UVA = univariate analysis; WBRT = whole-brain radiotherapy.

SUBMITTED August 18, 2014. ACCEPTED December 30, 2014.

INCLUDE WHEN CITING Published online July 3, 2015; DOI: 10.3171/2014.12.JNS141919.

DISCLOSURE The authors report no conflict of interest concerning the materials or methods used in this study or the findings specified in this paper.

* Dr. Frakes and Mr. Figura have contributed equally to this work. Drs. Rao and Etame share senior authorship of this work. 
$\mathrm{M}$ ALIGNANT melanoma is a leading cause of brain metastases after lung and breast cancers. Patients who harbor brain metastases uniformly demonstrate poorer outcomes than patients without brain metastases. ${ }^{15}$ Furthermore, the outcomes are much worse for those with untreated brain metastases than for patients who have radiotherapeutically controlled intracranial disease. ${ }^{15}$ Accordingly, radiotherapy plays a critical role in the management of melanoma brain metastases. Advances in immunotherapy and targeted therapies have led to enhanced disease response in and survival of patients with systemic metastases from malignant melanoma. ${ }^{13}$ Therefore, radiotherapy strategies that minimize intracranial neurological morbidities are critical in improving outcomes for these patients.

Traditionally, whole-brain radiotherapy (WBRT) was the predominant treatment paradigm. However, some data have indicated that stereotactic radiosurgery (SRS) can be used to attain similar overall survival (OS) and duration of functional independence without WBRT for single or multiple lesions. ${ }^{8}$ One potential concern in using SRS to treat multiple lesions has been the risk of radiation necrosis. However, SRS has demonstrated efficacy in treating 1-4 brain metastases with acceptable toxicities in clinical trials. ${ }^{14}$ Moreover, a very small number of retrospective studies have successfully demonstrated the feasibility and safety of SRS in treating patients with more than 4 metastases in lieu of WBRT with acceptable toxicity. ${ }^{1,4,6,7,9,12}$ Hence, SRS could serve as an ideal alternative to WBRT that could attain the goals of both local disease control in the setting of multiple metastases and the avoidance of the neurocognitive toxicities often associated with WBRT.

There are very limited data on SRS treatment of patients with melanoma brain metastases consisting of multiple lesions ( $>5$ ). In addition, the current data have been largely based on Gamma Knife surgery. Therefore, we sought to determine the effectiveness of linear accelerator (LINAC)-based SRS in treating patients who presented with at least 5 melanoma brain metastases. The primary end point of our study was local control (LC), while secondary end points focused on toxicity, OS, and distant failures.

\section{Methods}

We performed a retrospective analysis of our SRS brain metastases database for the period between January 2008 and December 2012. Patients who had been treated with SRS in a single session for $\geq 5$ lesions identified on staging or surveillance Gd-enhanced MRI and who had an established diagnosis of systemic melanoma were selected for study inclusion. The majority of patients had been initially referred for SRS to $\leq 3$ lesions, but additional disease was found at the time of thin-slice MRI for treatment planning. These patients were treated with SRS for all gross disease because of their age, performance status, and preference. The SRS-treated patients with a cancer diagnosis other than melanoma, as well as those with fewer than 5 lesions, were excluded. This study was approved by the institutional review board.

\section{LINAC-Based SRS Technique}

Tumor was defined using Gd-enhanced MRI (Siemens Sonata, Siemens Medical Systems) with 1-mm slices for treatment planning purposes before the delivery of radiation. The MR image was coregistered and fused with CT scanning data (General Electric Medical System). The gross tumor volume (GTV) was designated to encompass the confines of the contrast-enhancing lesion. The majority of cases had a uniform 1- to 2-mm expansion of the GTV to create the planning target volume (PTV). All patients were treated in a single fraction. Doses were prescribed to ensure at least 95\% coverage of the PTV with the prescription dose. Dose selection was based on previously published Radiation Therapy Oncology Group (RTOG) data, with dose modification left up to the treating physician. ${ }^{14}$ Dose de-escalation was based on proximity to critical structures or significant hemorrhage. Concurrent WBRT was also left up to the treating physician and was used in a minority of the patients. All gross disease was treated with SRS. Treatments were delivered using dynamic conformal arc radiotherapy. Patient immobilization was achieved using a commercially available head mask fixation system (BrainLab AG). Treatments were delivered with the BrainLab Novalis Classic LINAC with 6-MV photons. Imaging was provided with the BrainLab ExacTrac positioning system.

\section{Chart Review and Follow-Up}

We retrospectively reviewed patient charts for clinical parameters. In particular, we assessed demographic characteristics, tumor staging data, chemoradiation history, treatment course, imaging data, and SRS treatment plan. Whole-brain radiotherapy was defined as concurrent when, at the time of SRS, it was also planned; and it was defined as previous when it was performed at least 3 months prior to SRS. Patients were stratified based on the recursive partitioning analysis (RPA) classification system. All patients were followed up with clinical examinations and MRI at 2- to 3-month intervals performed in a clinic setting in which the treating radiation oncologists and neurosurgeons evaluated the patient together. Neurological status as well as treatment-related toxicity as defined by the RTOG grading system was assessed. Follow-up MRI studies were reviewed in conjunction with a neuroradiologist. In particular, we assessed for local failure and/ or radiation-induced changes. The most recent Response Evaluation Criteria in Solid Tumors (RECIST) were used to assess local failure based on each individually treated lesion. ${ }^{16}$ These criteria establish parameters for complete response, partial response, stable disease, and failure. Complete response, partial response, and stable disease were all considered satisfactory local disease control. Distant failure was defined as new brain metastases or leptomeningeal enhancement outside the previously irradiated volume. Given the inherent 2D limitations of RECIST, we also looked at the 3D volume-based response in patients who were close to the $20 \%$ cutoff for failures based on RECIST. ${ }^{2}$ This did not change the interpretations of the response, so only RECIST was included in this analysis. A multidisciplinary team including the treating neurosurgeon, radiation oncologist, pathologist, and neuroradiolo- 
gist assessed radionecrosis of the brain by using MRI. The primary end point for this study was local failure. Secondary end points included distant failure, OS, and toxicity of treatment calculated from the date of treatment to the event date.

\section{Statistical Analysis}

Statistical analyses were performed using JMP 11 (SAS Institute Inc.). Descriptive statistics were used to summarize the cohort, including the median and range for continuous variables or counts and percentages for categorical variables. The OS, LC, and distant control (DC) rates were calculated from the date of treatment to the date of death or progression by using the Kaplan-Meier (KM) method. The log-rank test was used to test differences between groups. The Cox proportional-hazards model was used for univariate and multivariate analysis (UVA and MVA, respectively) to assess the effect of patient, tumor, and other predictive factors of significance on the end points. A 2-tailed $p<0.05$ was considered statistically significant.

\section{Results}

\section{Patient Characteristics and Treatment Parameters}

We identified 149 metastatic brain lesions treated in 28 patients. The clinical characteristics of all 28 patients are listed in Table 1. The median patient age was 60.5 years (range 38-83 years). All patients had melanoma tumor histology. Twenty-four patients $(85.7 \%)$ had extracranial disease as well. The majority of patients $(67.8 \%)$ had 5 lesions, and the rest (32.1\%) had 6 lesions. All patients underwent LINAC-based single-fraction SRS. Four patients (14.3\%) had received previous WBRT, and 11 (39.3\%) had undergone previous SRS. The median radiation dose to each tumor was 24 Gy (range 15-24 Gy; Table 2). The median PTV was $0.34 \mathrm{~cm}^{3}$ (range $0.01-12.5 \mathrm{~cm}^{3}$ ). The median cumulative PTV for all lesions treated per patient was $3.7 \mathrm{~cm}^{3}$ (range $0.6-16.9 \mathrm{~cm}^{3}$ ). At the time of treatment, $7 \%$ of patients were categorized as RPA Class I, $89 \%$ as RPA Class II, and $4 \%$ as RPA Class III.

\section{Local and Distant Control}

The median follow-up for all patients was 6.3 months (range 1-46 months). The rate of local failure was $11.4 \%$. Kaplan-Meier estimates for LC at 6 and 12 months were $91.3 \%$ and $82.2 \%$, respectively (Fig. 1). Figure 2 displays a competing risk analysis of LC with patient survival. Results of MVA of LC and DC are displayed in Tables 3 and 4. On UVA and MVA a PTV $\geq 0.34 \mathrm{~cm}^{3}$ was a significant predictor of local failure with a HR $16.1(95 \% \mathrm{CI}$ 3.2-292.6, p < 0.0001) and HR 14.8 (95\% CI 3.0-268.4, p $=0.0002$ ), respectively. On UVA PTV $\geq 2 \mathrm{~cm}^{3}$ was a significant predictor of local failure with a HR of $3.76(95 \%$ CI $1.17-10.4, \mathrm{p}=0.03$ ); however, this factor did not show significance on MVA.

Sixteen patients (57.1\%) were noted to have distant failure in the brain with a median time to failure of 3 months (range 1-15 months). Nine patients with distant failures received WBRT, and 7 received additional SRS.
TABLE 1. Summary of characteristics in 28 patients with multiple melanoma metastases

\begin{tabular}{|c|c|}
\hline Variable & No. $(\%)$ \\
\hline No. of patients & 28 \\
\hline No. of lesions & 149 \\
\hline No. of patients w/ 5 lesions & $19(67.8)$ \\
\hline No. of patients w/ 6 lesions & $9(32.1)$ \\
\hline \multicolumn{2}{|l|}{ FU (mos) } \\
\hline Median & 6.25 \\
\hline Range & $1-46$ \\
\hline \multicolumn{2}{|l|}{ Age (yrs) } \\
\hline Median & 60.5 \\
\hline Range & $38-83$ \\
\hline \multicolumn{2}{|l|}{ Sex } \\
\hline $\mathrm{M}$ & $19(67.9)$ \\
\hline $\mathrm{F}$ & $9(32.1)$ \\
\hline \multicolumn{2}{|l|}{ KPS \% } \\
\hline 100 & $13(46.4)$ \\
\hline 90 & $8(28.6)$ \\
\hline 80 & $5(17.9)$ \\
\hline 70 & $1(3.6)$ \\
\hline 60 & $0(0)$ \\
\hline 50 & $1(3.6)$ \\
\hline \multicolumn{2}{|l|}{ RPA class } \\
\hline I & $2(7.1)$ \\
\hline II & $25(89.3)$ \\
\hline III & $1(3.6)$ \\
\hline \multicolumn{2}{|l|}{ Extracranial metastases } \\
\hline Present & $24(85.7)$ \\
\hline Absent & $4(14.3)$ \\
\hline \multicolumn{2}{|l|}{ Previous WBRT } \\
\hline Yes & $4(14.3)$ \\
\hline No & $24(85.7)$ \\
\hline \multicolumn{2}{|l|}{ Previous SRS or FSRT } \\
\hline Yes & $11(39.3)$ \\
\hline No & $17(60.7)$ \\
\hline \multicolumn{2}{|l|}{ Concurrent WBRT } \\
\hline Yes & $6(21.4)$ \\
\hline No & $22(78.6)$ \\
\hline \multicolumn{2}{|l|}{ First treatment after SRS } \\
\hline None & $12(42.9)$ \\
\hline WBRT & $9(32.1)$ \\
\hline SRS & $6(21.4)$ \\
\hline FSRT & $1(3.6)$ \\
\hline \multicolumn{2}{|l|}{ Total PTV $\left(\mathrm{cm}^{3}\right)$} \\
\hline Median & 3.7 \\
\hline Range & $0.6-16.9$ \\
\hline
\end{tabular}

FU = follow-up; KPS = Karnofsky Performance Scale; FSRT = fractionated stereotactic radiotherapy. 
TABLE 2. Summary of treatment characteristics for 149 metastatic lesions

\begin{tabular}{|c|c|}
\hline Variable & No. $(\%)$ \\
\hline \multicolumn{2}{|l|}{ PTV $\left(\mathrm{cm}^{3}\right)$} \\
\hline Median & 0.34 \\
\hline Range & $0.014-12.51$ \\
\hline \multicolumn{2}{|l|}{ GTV $\left(\mathrm{cm}^{3}\right)$} \\
\hline Median & 0.16 \\
\hline Range & $0.005-9.397$ \\
\hline \multicolumn{2}{|l|}{ Diameter (mm) } \\
\hline Median & 6.8 \\
\hline Range & $0.8-36$ \\
\hline \multicolumn{2}{|l|}{ Dose (Gy) } \\
\hline 15 & $1(0.7)$ \\
\hline 16 & $4(2.7)$ \\
\hline 18 & $10(6.7)$ \\
\hline 20 & $6(4.0)$ \\
\hline 21 & $45(30.2)$ \\
\hline 24 & $83(55.7)$ \\
\hline \multicolumn{2}{|l|}{ Local failure } \\
\hline Yes & $17(11.4)$ \\
\hline No & $110(73.8)$ \\
\hline No FU & $22(14.8)$ \\
\hline \multicolumn{2}{|c|}{ Time to local failure (mos) } \\
\hline Median & 5 \\
\hline Range & $2.8-46$ \\
\hline \multicolumn{2}{|c|}{ Location of metastases } \\
\hline Brainstem & $3(2.0)$ \\
\hline Cerebellum & $11(7.4)$ \\
\hline Frontal & $55(36.9)$ \\
\hline Frontal temporal & $1(0.7)$ \\
\hline Insular cortex & $2(1.3)$ \\
\hline Occipital & $15(10.1)$ \\
\hline Paraventricular & $2(1.3)$ \\
\hline Parietal & $36(24.2)$ \\
\hline Parietal occipital & $3(2.0)$ \\
\hline Temporal & $20(13.4)$ \\
\hline Thalamus & $1(0.7)$ \\
\hline
\end{tabular}

\section{Overall Survival}

The median OS was 9.4 and 7.6 months from the date of brain metastases diagnosis and the date of SRS, respectively. The KM OS estimate at 6 and 12 months was $57.8 \%$ and $28.2 \%$, respectively, from the time of SRS treatment. The RPA class was a significant predictor of KM OS estimates from the date of treatment $(p=0.02)$. Overall survival according to RPA class is shown in Fig. 3. Patients who did not receive WBRT after SRS treatment had decreased OS on MVA (HR 3.5, 95\% CI 1.1-12.0, p = 0.03), and patients who did not receive WBRT prior to SRS had improved OS (HR 0.11, 95\% CI 0.02-0.53, p = 0.007). Univariate and multivariate analyses for OS are shown in Table 5.

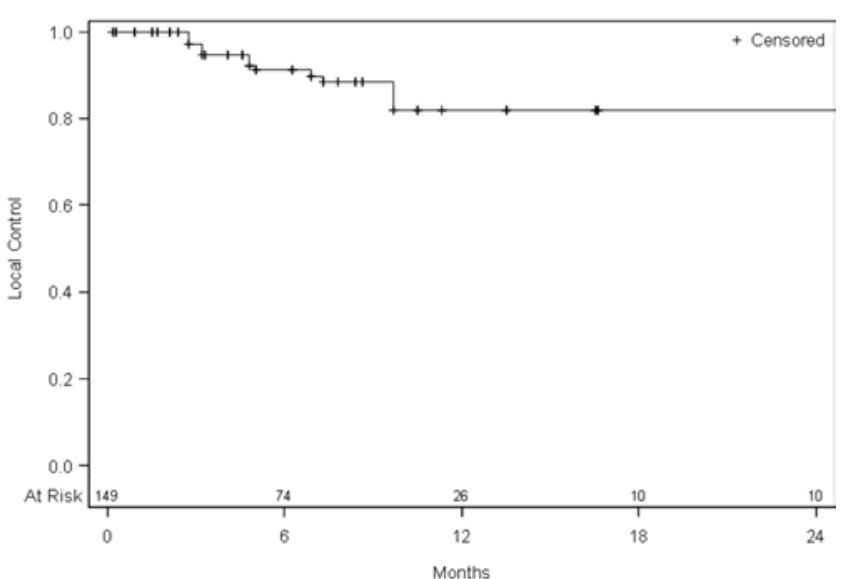

FIG. 1. Kaplan-Meier curve for LC.

\section{Toxicity}

Radiation necrosis was confirmed by histological resection in 1 patient (3\%). Otherwise, there was no acute or late RTOG Grade 3 or higher toxicity.

\section{Discussion}

Malignant melanoma has a predilection for the development of brain metastases. Interval development of brain metastases is an ominous sign with a grave prognosis. Although most patients with malignant melanoma are more likely to succumb to systemic complications, uncontrolled intracranial metastases can lead to significant neurological morbidity and death. Whole-brain radiotherapy has demonstrated effectiveness in controlling brain metastases but comes at a price since some patients will develop neurocognitive deficits posttreatment. ${ }^{10}$ Stereotactic radiosurgery is a focal strategy shown to be quite effective in treating melanoma metastases. ${ }^{11}$ However, there are very limited data on exploring its utility in treating patients with more than 4 lesions. ${ }^{1,4,6,7,9,12}$ Over the last few years,

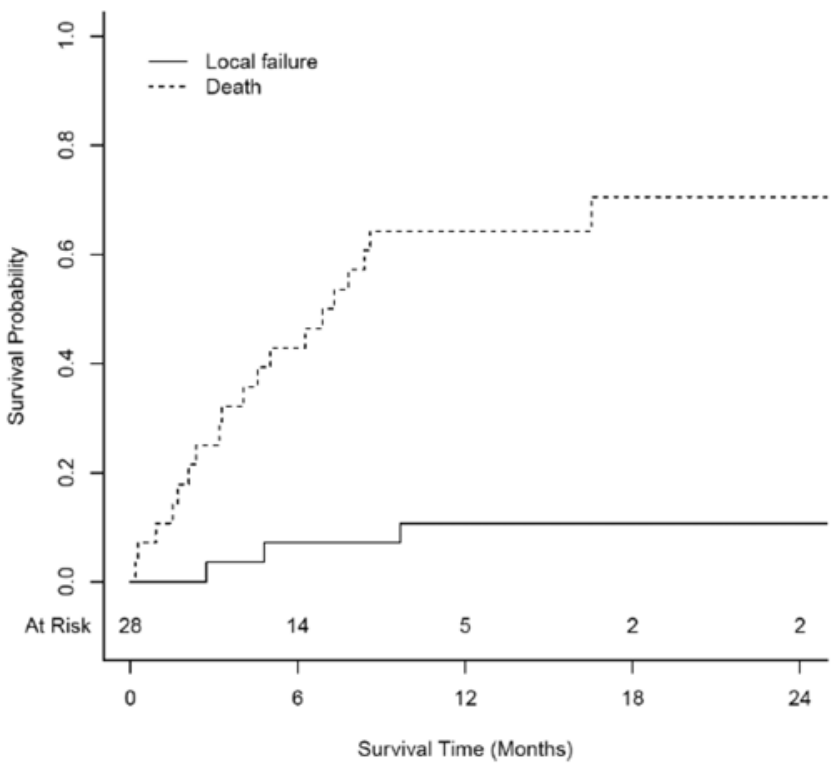

FIG. 2. Competing risk analysis of $L C$ with patient survival. 
TABLE 3. Univariate and multivariate analysis of local control

\begin{tabular}{ccccc}
\hline Analysis & HR & $p$ Value & $95 \% \mathrm{Cl}$ & Comparison Group \\
\hline Univariate & & & & \\
\hline PTV vol: $\geq 0.34 \mathrm{~cm}^{3}$ & 16.14 & $<0.0001$ & $3.24-292.56$ & $<0.34 \mathrm{~cm}^{3}$ \\
\hline GTV vol: $\geq 0.16 \mathrm{~cm}^{3}$ & 4.95 & 0.0045 & $1.59-21.60$ & $<0.16 \mathrm{~cm}^{3}$ \\
\hline Dose: $<24$ Gy & 2.5 & 0.066 & $0.94-7.33$ & $\geq 24$ Gy \\
\hline Previous WBRT: no & 0.77 & 0.75 & $0.077-7.48$ & Yes \\
\hline Concurrent WBRT: no & 0.69 & 0.55 & $0.39-4.42$ & $<2 \mathrm{~cm}^{3}$ \\
\hline PTV vol: $\geq 2 \mathrm{~cm}^{3}$ & 3.76 & 0.028 & $1.17-10.4$ & $<3.7 \mathrm{~cm}^{3}$ \\
\hline Total PTV vol: $\geq 3.7 \mathrm{~cm}^{3}$ & 9.96 & 0.003 & $1.91-182.71$ & $<0.34 \mathrm{~cm}^{3}$ \\
\hline Multivariate & & & & $<0.16 \mathrm{~cm}^{3}$ \\
\hline PTV vol: $\geq 0.34 \mathrm{~cm}^{3}$ & 14.79 & 0.0002 & $2.95-268.45$ & $<2 \mathrm{~cm}^{3}$ \\
\hline GTV vol: $\geq 0.16 \mathrm{~cm}^{3}$ & 4.06 & 0.0194 & $1.24-18.25$ & $<3.7 \mathrm{~cm}^{3}$ \\
\hline PTV vol: $\geq 2 \mathrm{~cm}^{3}$ & 2.65 & $<0.12$ & $0.75-8.92$ & $1.87-181.58$ \\
\hline Total PTV vol: $\geq 3.7 \mathrm{~cm}^{3}$ & 9.87 & 0.004 & & \\
\hline
\end{tabular}

we have made a paradigm shift within our radiotherapy practice whereby SRS has become the preeminent treatment modality for patients with multiple brain metastases, and melanoma has been the predominant tissue histology. We were therefore in a unique position to assess the effectiveness and safety of SRS in patients with 5 or more melanoma brain metastases given the intrinsic radioresistant phenotype of melanoma. Our results clearly demonstrate acceptable LC rates without any significant toxic effects when SRS is employed in melanoma patients with 5 or more brain metastases.

The ability to locally control melanoma brain metastases with LINAC-based SRS was the primary outcome objective in our study. Our findings of excellent LC rates were consistent with prior reports in the literature..$^{1,4,12}$ In particular, we noted a local failure rate of $11.4 \%$. The KM LC estimates at 6 and 12 months were $91.3 \%$ and $82.2 \%$, respectively. Salvetti et al. reported similar control rates of $92.4 \%$ at 6 months and $84.8 \%$ at 12 months when they used Gamma Knife radiosurgery to treat 5 or more brain metastases..$^{2}$ Our results at least demonstrate effective control rates with SRS even for radioresistant tumors like melanoma. More importantly, we identify a potential role for SRS in the LC of multiple brain metastases from malignant melanoma.

In addition, our study shares features similar to those in previous series looking at SRS for more than 4 lesions. ${ }^{1,4,6,7,9,12}$ Table 5 summarizes results of the current study, which are compared with previous published data on treating multiple brain metastases ( $\geq 5$ lesions). As in the above series, our patient population was significantly enriched with RPA Class II patients. At the time of treatment, $7 \%$ of patients were classified as RPA Class I, $89 \%$ as RPA Class II, and $4 \%$ as RPA Class III. The RPA class is particularly important since it is prognostic for OS. ${ }^{3}$ Our median PTV $\left(0.34 \mathrm{~cm}^{3}\right.$, range $\left.0.01-12.5 \mathrm{~cm}^{3}\right)$ was much smaller than the median PTV from prior studies (range $\left.1.2-10.9 \mathrm{~cm}^{3}\right){ }^{1,4,6,7,9,12}$ This difference is a reflection of the fact that melanoma patients at our center underwent routine surveillance brain MRI every 2-3 months. Hence, lesions were usually identified and treated very early on in the course of brain metastases. Since there is an established inverse correlation between tumor size and LC from SRS, ${ }^{5,14}$ our surveillance strategy could account for the excellent LC rates we observe for radioresistant metastases such as malignant melanoma.

A significant predictor of treatment failure was the PTV volume. We noted that a PTV volume $\geq 0.34 \mathrm{~cm}^{3}$ was a significant predictor of local failure on UVA (HR 16.1, 95\% CI 3.2-292.6; $\mathrm{p}<0.0001$ ) and MVA (HR 14.8, 95\% CI 3.0-268.5, $\mathrm{p}=0.0002)$. A growing body of literature uses a PTV cutoff of $2 \mathrm{~cm}^{3}$ as significant for improved LC, which was less significant in our cohort. ${ }^{17}$ As previously mentioned, our PTV volumes were markedly smaller than the median PTVs of similar series for the reasons described above. While LC was excellent, distant failures within the brain were noted for 16 patients $(57.1 \%)$, a reflection of the aggressive biology of malignant melanoma.

TABLE 4. Univariate and multivariate analysis of distant control

\begin{tabular}{cllll}
\hline Analysis & HR & $p$ Value & $95 \% \mathrm{Cl}$ & Comparison Group \\
\hline Univariate & & & & Yes \\
\hline Previous WBRT: no & 0.51 & 0.43 & $0.13-3.35$ & Yes \\
\hline Concurrent WBRT: no & 5.13 & 0.049 & $1.01-93.6$ & Yes \\
\hline Primary controlled: no & 0.68 & 0.47 & $0.51-4.51$ & $<3.7 \mathrm{~cm}^{3}$ \\
\hline Total PTV vol: $\geq 3.7 \mathrm{~cm}^{3}$ & 1.2 & 0.37 & $0.37-3.91$ & Yes \\
\hline Multivariate & & & & $0.93-88.3$ \\
\hline Concurrent WBRT: no & 4.8 & 0.065 & &
\end{tabular}




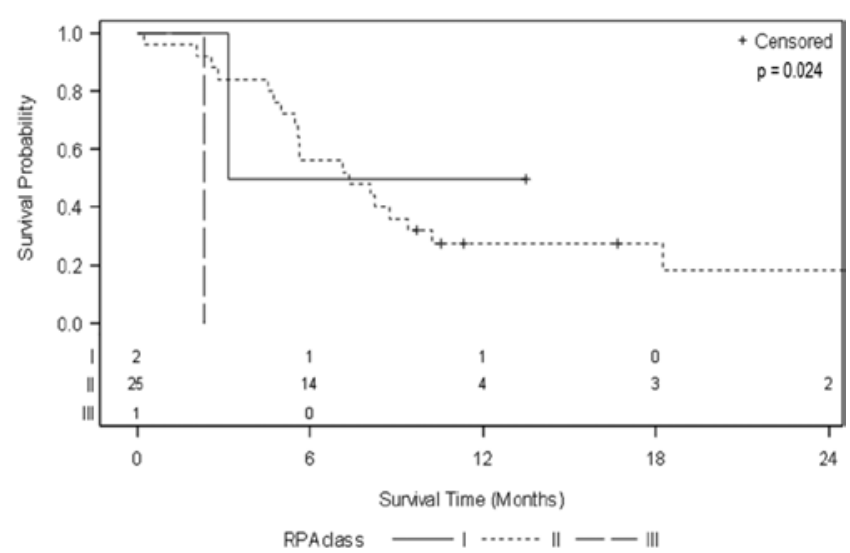

FIG. 3. Overall survival based on RPA class.

Since our practice paradigm favors SRS over WBRT, we were able to control distant failures in 7 of the 16 patients by using SRS. The remaining 9 patients were treated with WBRT as a last salvage measure. Since we are prone to identify and treat lesions with SRS when they are smaller, we can subsequently treat newer lesions with SRS when they arise given the low probability of SRS treatment plan overlap and delayed WBRT.

We observed median OSs of 9.4 and 7.6 months from the date of brain metastases diagnosis and the date of SRS, respectively. The KM OS estimate at 6 and 12 months was $57.8 \%$ and $28.2 \%$, respectively, from the time of SRS treatment. Our median OS falls within the higher range as compared with similar series. It is also excellent given that our series is enriched with the radioresistant histology of melanoma. ${ }^{1,4,6,7,9,12}$ The most recent multiinstitutional prospective observational study found a median OS of 10.8 months in patients with 5-10 lesions, which was comparable to survival in those with $2-4$ lesions. ${ }^{18}$ The population in that observational study is different from our cohort given that the majority of their patients had controlled extracerebral disease $(68 \%)$ and that all primary histolo- gies were included, with a minority of melanoma cases. We further identified RPA class as a significant predictor of KM OS estimates, a finding consistent with what has already been established. We also noted a paradoxical effect of WBRT on OS, in that OS was favorably impacted in patients who did not receive WBRT prior to SRS. This finding could be a reflection of the intracranial disease burden. Interestingly, OS was adversely impacted in patients who did not receive WBRT after SRS treatment. The reasons for such an observation are quite unclear. Our series contained only 28 patients with a total of 149 lesions. Perhaps additional studies with larger sample sizes could further elucidate the significance of the paradoxical effects of WBRT on OS in patients with melanoma.

Stereotactic radiosurgery for multiple melanoma brain metastases did not appear to induce any increase in significant toxicity either acute or delayed in our patient cohort. A larger sample size will be necessary for future studies, and the small sample is a limitation of our current analysis. Furthermore, our study has the inherent limitations of a retrospective study. Patients received varied systemic therapy for a variable disease burden at the time of SRS. Additionally, our patients had smaller PTV volumes than those previously published. Nonetheless, this study is unique in addressing the role of LINAC-based SRS in treating patients with 5-6 melanoma brain metastases.

\section{Conclusions}

In summary, this study provides retrospective outcomes from a single institution utilizing LINAC-based SRS in treating multiple melanoma brain metastases. We were able to achieve minimal toxicity while maintaining excellent local disease control for this radioresistant entity. Data in this study support a role for SRS in multiple brain metastases.

\section{References}

1. Bhatnagar AK, Flickinger JC, Kondziolka D, Lunsford LD:

TABLE 5. Univariate and multivariate analysis of overall survival from treatment date

\begin{tabular}{|c|c|c|c|c|}
\hline Analysis & $\mathrm{HR}$ & $p$ Value & $95 \% \mathrm{Cl}$ & Comparison Group \\
\hline \multicolumn{5}{|l|}{ Univariate } \\
\hline KPS score: $\leq 70$ & 2.12 & 0.360 & $0.13-3.00$ & $>70$ \\
\hline Primary disease: not controlled & 0.79 & 0.590 & $0.33-1.88$ & Controlled \\
\hline Age: $\geq 61$ yrs & 0.72 & 0.45 & $0.30-1.70$ & $<61 \mathrm{yrs}$ \\
\hline Extracranial metastases: present & 2.16 & 0.260 & $0.62-13.68$ & Absent \\
\hline RPA class: II & 1.4 & 0.73 & $0.29-25.19$ & I \\
\hline RPA class: III & 17.77 & 0.08 & $0.59-574.97$ & \\
\hline Previous WBRT: no & 0.21 & 0.022 & $0.07-0.78$ & Yes \\
\hline Concurrent WBRT: no & 0.69 & 0.49 & $0.267-2.127$ & Yes \\
\hline Post-WBRT: no & 2.53 & 0.04 & $1.04-6.76$ & Yes \\
\hline PTV vol: $\geq 2 \mathrm{~cm}^{3}$ & 0.74 & 0.43 & $0.31-1.48$ & $<2 \mathrm{~cm}^{3}$ \\
\hline Total PTV vol: $\geq 3.7 \mathrm{~cm}^{3}$ & 0.67 & 0.41 & $0.25-1.72$ & $<3.7 \mathrm{~cm}^{3}$ \\
\hline \multicolumn{5}{|l|}{ Multivariate } \\
\hline Previous WBRT: no & 0.11 & 0.007 & $0.021-0.53$ & Yes \\
\hline Post WBRT: no & 3.46 & 0.033 & $1.10-11.96$ & Yes \\
\hline
\end{tabular}


Stereotactic radiosurgery for four or more intracranial metastases. Int J Radiat Oncol Biol Phys 64:898-903, 2006

2. Follwell MJ, Khu KJ, Cheng L, Xu W, Mikulis DJ, Millar $\mathrm{BA}$, et al: Volume specific response criteria for brain metastases following salvage stereotactic radiosurgery and associated predictors of response. Acta Oncol 51:629-635, 2012

3. Gaspar LE, Scott C, Murray K, Curran W: Validation of the RTOG recursive partitioning analysis (RPA) classification for brain metastases. Int J Radiat Oncol Biol Phys 47:10011006,2000

4. Grandhi R, Kondziolka D, Panczykowski D, Monaco EA III, Kano H, Niranjan A, et al: Stereotactic radiosurgery using the Leksell Gamma Knife Perfexion unit in the management of patients with 10 or more brain metastases. J Neurosurg 117:237-245, 2012

5. Han JH, Kim DG, Chung HT, Paek SH, Park CK, Jung HW: Radiosurgery for large brain metastases. Int J Radiat Oncol Biol Phys 83:113-120, 2012

6. Hunter GK, Suh JH, Reuther AM, Vogelbaum MA, Barnett $\mathrm{GH}$, Angelov L, et al: Treatment of five or more brain metastases with stereotactic radiosurgery. Int J Radiat Oncol Biol Phys 83:1394-1398, 2012

7. Kim CH, Im YS, Nam DH, Park K, Kim JH, Lee JI: Gamma knife radiosurgery for ten or more brain metastases. J Korean Neurosurg Soc 44:358-363, 2008

8. Kocher M, Soffietti R, Abacioglu U, Villà S, Fauchon F, Baumert BG, et al: Adjuvant whole-brain radiotherapy versus observation after radiosurgery or surgical resection of one to three cerebral metastases: results of the EORTC 2295226001 study. J Clin Oncol 29:134-141, 2011

9. Lee CK, Lee SR, Cho JM, Yang KA, Kim SH: Therapeutic effect of gamma knife radiosurgery for multiple brain metastases. J Korean Neurosurg Soc 50:179-184, 2011

10. Li J, Bentzen SM, Li J, Renschler M, Mehta MP: Relationship between neurocognitive function and quality of life after whole-brain radiotherapy in patients with brain metastasis. Int J Radiat Oncol Biol Phys 71:64-70, 2008

11. Mori Y, Kondziolka D, Flickinger JC, Kirkwood JM, Agarwala S, Lunsford LD: Stereotactic radiosurgery for cerebral metastatic melanoma: factors affecting local disease control and survival. Int J Radiat Oncol Biol Phys 42:581-589, 1998

12. Salvetti DJ, Nagaraja TG, McNeill IT, Xu Z, Sheehan J: Gamma Knife surgery for the treatment of 5 to 15 metastases to the brain: clinical article. J Neurosurg 118:1250-1257, 2013

13. Saraceni MM, Khushalani NI, Jarkowski A III: Immunotherapy in melanoma: recent advances and promising new therapies. J Pharm Pract [epub ahead of print], 2014
14. Shaw E, Scott C, Souhami L, Dinapoli R, Kline R, Loeffler J, et al: Single dose radiosurgical treatment of recurrent previously irradiated primary brain tumors and brain metastases: final report of RTOG protocol 90-05. Int J Radiat Oncol Biol Phys 47:291-298, 2000

15. Tsao MN, Lloyd N, Wong RK, Chow E, Rakovitch E, Laperriere $\mathrm{N}$, et al: Whole brain radiotherapy for the treatment of newly diagnosed multiple brain metastases. Cochrane Database Syst Rev 4:CD003869, 2012

16. van Persijn van Meerten EL, Gelderblom H, Bloem JL: RECIST revised: implications for the radiologist. A review article on the modified RECIST guideline. Eur Radiol 20:1456-1467, 2010

17. Varlotto JM, Flickinger JC, Niranjan A, Bhatnagar A, Kondziolka D, Lunsford LD: The impact of whole-brain radiation therapy on the long-term control and morbidity of patients surviving more than one year after gamma knife radiosurgery for brain metastases. Int J Radiat Oncol Biol Phys 62:1125-1132, 2005

18. Yamamoto M, Serizawa T, Shuto T, Akabane A, Higuchi Y, Kawagishi J, et al: Stereotactic radiosurgery for patients with multiple brain metastases (JLGK0901): a multi-institutional prospective observational study. Lancet Oncol 15:387-395, 2014

\section{Author Contributions}

Conception and design: Etame, Chinnaiyan, Rao. Acquisition of data: Frakes, Figura, Patel, Sarangkasiri. Analysis and interpretation of data: Etame, Frakes, Ahmed, Juan, Strom. Drafting the article: Etame, Frakes, Figura, Rao. Critically revising the article: Etame, Frakes, Figura, Ahmed, Juan, Latifi, Chinnaiyan, Rao. Reviewed submitted version of manuscript: all authors. Approved the final version of the manuscript on behalf of all authors: Etame. Statistical analysis: Ahmed, Juan, Strom.

\section{Supplemental Information}

\section{Previous Presentation}

Portions of this work were presented in poster form at the American Radium Society Annual Meeting held in St. Thomas, US Virgin Islands, on April 28, 2014.

\section{Correspondence}

Arnold B. Etame, H. Lee Moffitt Cancer Center and Research Institute, Department of Neuro-Oncology, 12902 Magnolia Dr., Tampa, FL 33612. email: arnold.etame@moffitt.org. 